\title{
Characterization of acrylic polyamide plastic embolization particles in vitro and in human tissue sections by light microscopy, infrared microspectroscopy and scanning electron microscopy with energy dispersive X-ray analysis
}

\author{
Linda A Murakata, Michael R Lewin-Smith, Charles S Specht, Victor F Kalasinsky, \\ Peter L McEvoy, Tuyethoa N Vinh, Lionel NMI Rabin and Florabel G Mullick
}

Armed Forces Institute of Pathology (AFIP), Washington, DC, USA

\begin{abstract}
Vascular embolization is a well-established practice for the treatment of tumors and vascular lesions. Rounded beads (microspheres) of various materials (collagen, dextran and trisacryl-polymer-gelatin) were developed to solve problems encountered with earlier versions of embolic material. We performed histochemistry, Fourier transform infrared microspectroscopy and scanning electron microscopy with energy dispersive X-ray analysis on two uterine and one hepatic specimen with unidentified intravascular foreign material, and examined a reference embolization product for comparison. The hematoxylin and eosin stained tissue sections showed multiple foci with unidentified intravascular foreign material and fibrous obliteration of vessel lumens. Only one case had a clinical history of previous embolization but without specifying the material used. One case was submitted for identification of a 'parasite'. The material stained positively with Sirius red and mucicarmine, variably with Masson's trichrome stain and Movat pentachrome, and did not stain centrally with periodic acid Schiff with diastase. Infrared spectrophotometric analysis of the material from all three cases demonstrated the spectrum of acrylic polyamide plastic. A control sample of EmboGold ${ }^{\mathrm{TM}}$ exhibited infrared microspectroscopic spectra similar to the three tissue specimens. Analysis by scanning electron microscopy with energy dispersive X-ray analysis demonstrated some differences in elemental composition between the tissue sections and the selected reference material. To our knowledge, this is the first report of infrared spectrophotometric analysis with scanning electron microscopy with energy dispersive $X$-ray analysis of an acrylic polyamide plastic embolization product both in vitro and in human histologic tissue sections. In cases lacking appropriate clinical information, identification by these methods and/or a panel of special stains may assist pathologists unfamiliar with this material's light microscopic appearance.
\end{abstract}

Modern Pathology (2006) 19, 922-930. doi:10.1038/modpathol.3800595; published online 14 April 2006

Keywords: embolization; uterus; fibroids; tumors; microspheres; Embosphere ${ }^{\circledR}$

Iatrogenic arterial embolization has been used as a nonsurgical technique for the treatment of tumors and vascular malformations. ${ }^{1}$ In primary hepatocellular carcinoma, surgery is the method

Correspondence: Dr LA Murakata, MD, Department of Environmental and Infectious Disease Sciences, Armed Forces Institute of Pathology, 6825 16th Street, NW, Washington, DC 20306-6000, USA.

E-mail: Murakata@afip.osd.mil

Received 17 January 2006; revised and accepted 27 February 2006; published online 14 April 2006 most likely to result in a cure. However, by the time the tumor is diagnosed, it is frequently either too extensive or the underlying liver disease is too severe for a surgical cure. A variety of methods have been used to treat inoperable hepatocellular carcinoma, but no one method has proven to be superior to the others. A minimally invasive treatment, trans-arterial hepatic chemoembolization, has been used for both primary liver tumors and metastatic lesions to the liver. Embolization microspheres are occlusive agents composed of both resorbable and nonresorbable materials, including 
polyvinyl alcohol, chitosan-coated alginate, degradable starch, aromatic oil gelatin, ethyl cellulose, albumin, dextran, glass, wax, silicone, polystyrene and polyacrylates. ${ }^{1-5}$

Uterine artery embolization is a nonsurgical treatment that preserves the uterus and offers an alternative to surgical procedures such as hysterectomy and myomectomy. Uterine artery embolization has been used as primary therapy for fibroids since $1995 .{ }^{6}$ This treatment has been shown to control both menorrhagia and symptoms caused by the bulk of fibroids in $85-90 \%$ of patients. ${ }^{7,8}$ Complications are rare and include post-treatment sepsis, late infection of the endometrium or myometrium, severe ischemic injury to the uterus (1-2\%), spontaneous passage of fibroid tissue and amenorrhea. ${ }^{7,8}$ Initially, polyvinyl alcohol particles were used as the embolic agent, injected through catheters placed in the uterine arteries. There were good results for symptom improvement, ${ }^{9-11}$ but mechanical drawbacks were reported. These included the irregularly shaped polyvinyl alcohol particles tending to aggregate and clump both within the catheter and inside the vessel more proximally than intended, leading to limited penetration. ${ }^{12}$ Subsequently, spherical embolic agents were developed made of various resorbable and nonresorbable materials (collagen, dextran and trisacryl polymer with gelatin). ${ }^{1,13}$

Embosphere ${ }^{\circledR}$ Microspheres and EmboGold ${ }^{\mathrm{TM}}$ are produced by Biosphere Medical $^{\mathrm{TM}}$ Inc. and are reportedly composed of a trisacryl-co-polymer crosslinked with gelatin. The microspheres are made of clear acrylic and were previously used as a microcarrier for cell culture. Positive characteristics reportedly include compressibility, easier passage through microcatheters with a luminal diameter smaller than that of the spheres, uniformity in size, no size change in liquids, little tendency to clump after injection and less tissue reaction in animal studies than with polyvinyl alcohol. $^{13,14}$

Marketing of this product began in Europe in 1994. In the United States, the Food and Drug Administration (FDA) initially cleared Embosphere ${ }^{\circledR}$ Microspheres in April, 2000, for treatment of hypervascular tumors and arteriovenous malformations. ${ }^{1,15}$ Clinical trials of Embosphere ${ }^{\circledR}$ Microspheres for uterine fibroid embolization have been ongoing in the US since May 2000, resulting in FDA clearance for this usage in November 2002. ${ }^{15}$ EmboGold $^{\mathrm{TM}}$ Microspheres (a colored version of Embosphere ${ }^{\circledR}$ Microspheres) was cleared by the FDA for treatment of hypervascular tumors and arteriovenous malformations, but this version is not approved for uterine artery embolization (EmboGold $^{\mathrm{TM}}$ product insert).

We received three specimens for identification of either 'foreign material' and/or a 'parasite'. In all three cases, we identified the material as acrylicpolyamide plastic consistent with that used to make the Embosphere ${ }^{\circledR}$ Microsphere embolization products. In view of the relative lack of published data on the in vivo characteristics of embolization products, we are reporting the in vivo histology, scanning electron microscopy and energy dispersive $\mathrm{X}$-ray analysis findings, and infrared spectrophotometric analysis of embolic microspheres in two hysterectomy specimens and one hepatic segmentectomy specimen. Although the product employed in these three cases is unknown, for comparison, scanning electron microscopy with energy dispersive X-ray analysis and infrared spectroscopic characteristics of an authentic sample of an acrylic polyamide plastic embolization product, EmboGold $^{\mathrm{TM}}$ (Biosphere Medical, Paris, France), are also reported.

Case 1: A 45-year-old female had undergone uterine artery embolization 2 years previously for irregular bleeding. Recent examination and ultrasound showed a 20-week-sized uterus and multiple fibroids. She had an elective hysterectomy because of the significant uterine size and failure of previous treatment. 'Foreign' material was noted in the hematoxylin and eosin stained sections by the contributor, who requested that we identify the material and rule out a parasitic infection.

Case 2: A 43-year-old morbidly obese female was undergoing a duodenal 'switch' procedure when it was noted that her uterus was enlarged (643 gm), and the surgeon performed a supracervical hysterectomy. Her past medical history was unremarkable except for tubal ligation 7 years prior to hysterectomy. The hematoxylin and eosin sections of the uterus showed extensive adenomyosis, but did not contain fibroids. In addition, an amorphous 'blue material' was seen in blood vessels that elicited a foreign body reaction, and the case was submitted for identification of the 'foreign material'. No history of previous uterine artery embolization was provided.

Case 3: A 64-year-old man with a liver mass and no known risk factors was found to have hepatocellular carcinoma on liver biopsy. A left lateral segmentectomy was performed and the specimen contained, in addition to hepatocellular carcinoma, amorphous material surrounded by multinucleated giant cells. We received the specimen for identification of the amorphous material. The clinical history did not mention a previous tumor embolization procedure.

\section{Materials and methods}

Hematoxylin and eosin stained slides from a hepatic segmentectomy specimen and two hysterectomy specimens were examined by light microscopy. Additional stains performed included Periodic acid Schiff with diastase, mucicarmine, Masson's trichrome, $72 \mathrm{~h}$ Oil red O, Movat, acid mucopolysaccharide and Sirius red for amyloid (a modified 
cotton dye). Staining method details are given in the Armed Forces Institute of Pathology's 'Laboratory Methods in Histochemistry'. ${ }^{16}$

In addition, tissue specimens approximately $5 \mu \mathrm{m}$ thick were cut from paraffin-embedded formalinfixed tissue, and then mounted on aluminum-coated glass slides for infrared spectroscopy or on carbon disks for scanning electron microscopy. No conductive material was coated onto the specimens for the scanning electron microscopy.

As no information was provided as to the embolization product employed in any of the three cases, we elected to examine a sample of embolization microspheres reportedly composed of trisacrylco-polymer crosslinked with gelatin. A sample of EmboGold ${ }^{\mathrm{TM}}$ Microspheres (Biosphere Medical, SA, Paris, France) embolization microspheres was selected as a representative referent material for spectroscopic comparison. The EmboGold ${ }^{\mathrm{TM}}$ sample examined was received in a prefilled $20 \mathrm{ml}$ syringe containing apyrogenic, sterile, physiological saline. The EmboGold ${ }^{\mathrm{TM}}$ microspheres were glued onto a carbon disk with Duco Cement ${ }^{\mathbb{B}}$ (Devcon $^{\mathrm{TM}}$ Riviera Beach, FL, USA), or cut in half and flattened onto an aluminum-coated slide.

Infrared spectra were obtained by using a Nicolet Continuum infrared microscope attached to a Nicolet model 860 Fourier transform infrared spectrometer (ThermoNicolet, Madison, WI, USA). A reflective Cassagrainian objective $(\times 32)$ was used to focus the infrared light onto tissue sections, which were mounted on aluminum-coated glass slides. The infrared spectrometer was operated in the 'reflection' mode wherein the light passed through the sample, reflected off the aluminum surface, and passed through the sample a second time before being directed to a liquid-nitrogencooled mercury-cadmium-telluride detector with a $0.25-\mathrm{mm}$ square element. Spectra of the specimens were ratioed to background spectra obtained from blank regions on the aluminum-coated slide, and adjustable apertures (between 20 and $50 \mu \mathrm{m})$ were used to direct the infrared beam to the areas of interest. Spectra were recorded at $4-\mathrm{cm}^{-1}$ spectral resolution, and 128 scans were co-added to reduce noise levels and provide adequate spectral quality. The measured infrared spectra were compared with those of the authentic EmboGold ${ }^{\mathrm{TM}}$ sample and to spectra stored in a digital spectral library. The spectrometer was purged with dry air to minimize the amounts of atmospheric water vapor and carbon dioxide in the spectrometer.

A Hitachi model S-3500N scanning electron microscope (Hitachi Instruments, Inc., San Jose, CA, USA) equipped with a ThermoNoran (ThermoNoran, Madison, WI, USA) energy-dispersive X-ray spectroscopy accessory was used to determine the elemental composition of the materials in the tissue. The electron beam was produced with a $15-\mathrm{kV}$ accelerating voltage and was directed at the speci- mens mounted on carbon disks. X-rays produced by the elements in the specimen were directed to the liquid-nitrogen-cooled silicon detector fitted with a Norvar ${ }^{\circledR}$ window, which allowed the identification of elements with atomic numbers as low as that of beryllium on the basis of their characteristic X-ray energies.

\section{Results}

In vitro hematoxylin and eosin histology of the reference sample of EmboGold ${ }^{\mathrm{TM}}$ Microspheres shows shatter artifact, "Venetian blind effect' ${ }^{17}$ of the plastic beads and has a similar appearance to that seen in the three resected specimens (Figure 1). The results of the histochemical stains performed on the reference sample and all three cases are shown in Table 1. The positively stained Sirius red material in the tissue and reference demonstrated partial birefringence when viewed under polarized light. Birefringence was not noted in any other stained sections.

Scanning electron microscopy shows some shrinkage artifact after the material was dried and flattened (Figure 2). The reference sample microspheres were listed as having diameters in the range of $500-700 \mu \mathrm{m}$, but scanning electron microscopy showed microspheres that ranged in size from 250$350 \mu \mathrm{m}$ (this is presumably due to drying). Scanning electron microscopy with energy dispersive X-ray analysis demonstrated peaks for carbon and oxygen with small peaks for sodium, chlorine and gold (Figure 2). Scanning electron microscopy with energy dispersive X-ray analysis of the material in the tissue showed peaks for carbon, oxygen, phosphorus and calcium.

Figure 3 shows the infrared spectra of the reference material and the three cases. All three cases have similar spectral peaks that are consistent with an acrylic polyamide plastic, and also closely match the spectrum obtained from EmboGold ${ }^{\mathrm{TM}}$ Microspheres, which are described as being composed of trisacryl-co-polymer crosslinked with gelatin.

Histologically, both hysterectomy specimens showed multiple obliterated vessels with lumenal fibrosis and occlusion. In addition, Case 1 showed multiple viable and hyalinized leiomyomas with occasional dystrophic calcification, and Case 2 had extensive adenomyosis, but no leiomyomas were identified. The fibrous tissue in the obliterated vessel lumen contained rounded spaces, singly and clustered, that were occasionally empty, but otherwise contained rounded focally distorted nonbirefringent particles of variably eosinophilic to basophilic material, which lacked internal structures. A 'Venetian blind' or 'fish scale' appearance that was favored to reflect a cutting artifact was also noted. ${ }^{17}$ Towards the margin of the spaces and within the adjacent fibrous tissue were various 
a
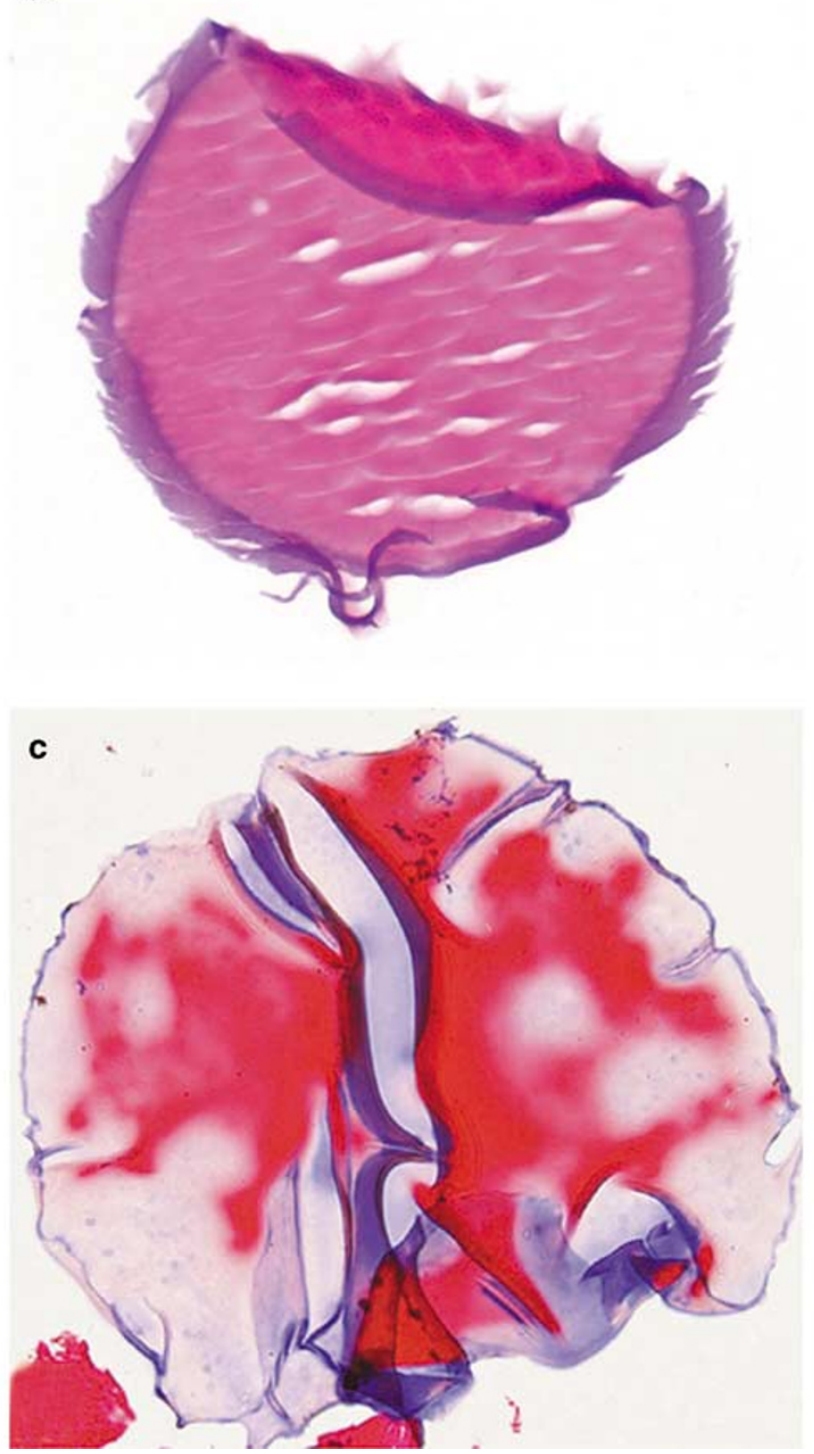

b

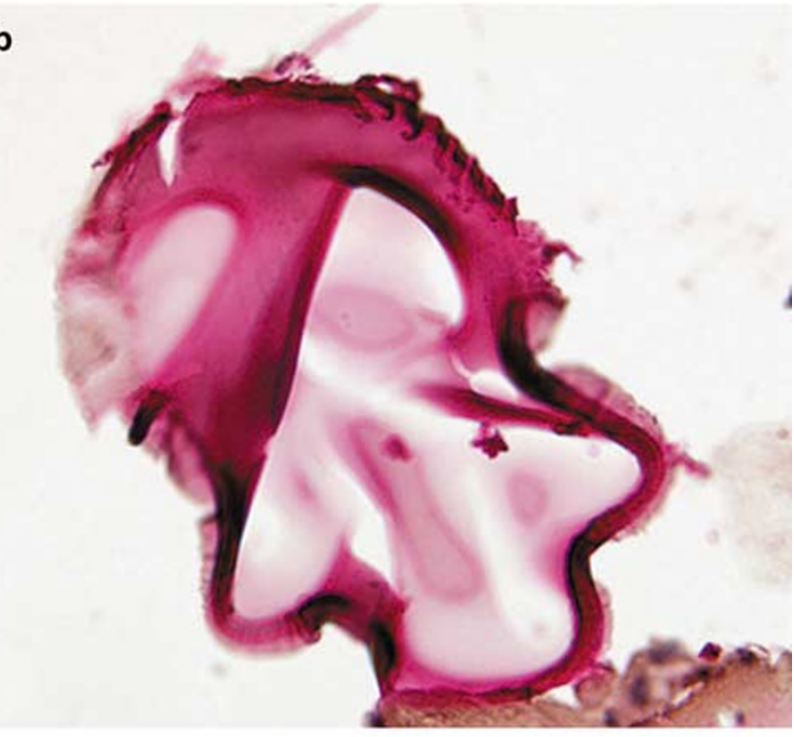

d

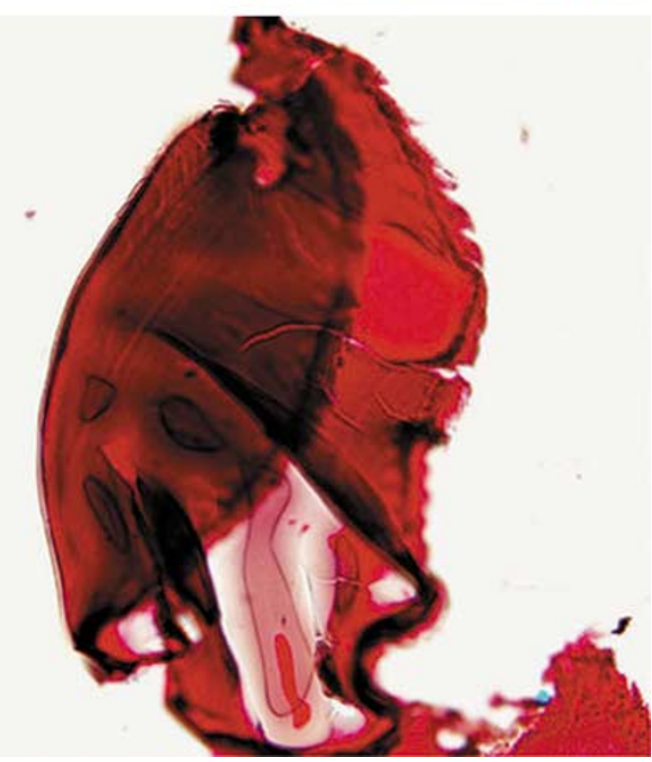

Figure 1 EmboGold ${ }^{\mathrm{TM}}$ Microspheres, histology. In vitro histology shows shatter 'Venetian blind effect' of plastic beads. (a) Hematoxylin and eosin stain. (B) Mucicarmine-positive (magenta). (c) Masson's trichrome stain-variable (blue/red). (d) Movat pentachrome stain-red/ black $(\times 100)$.

Table 1 Histochemical stain results

\begin{tabular}{|c|c|c|c|c|c|c|c|}
\hline Case no. & $\begin{array}{l}\text { Acid } \\
\text { mucopolysaccharide } \\
(A M P)\end{array}$ & Sirius red & $P A S / D$ & Masson's trichrome & $\begin{array}{l}\text { Movat } \\
\text { pentachrome }\end{array}$ & Mucicarmine & Oil-red-O \\
\hline 1 & NEG (pink) & POS (red) & NEG (blue) ${ }^{\mathrm{a}}$ & Variably blue and red & Black/red & POS (magenta) & NEG \\
\hline 2 & NEG (pink) & POS (red) & NEG (blue) ${ }^{\mathrm{a}}$ & Variably blue and red & Black/red & POS (magenta) & NEG \\
\hline 3 & NEG (pink) & POS (red) & NEG (blue) ${ }^{\mathrm{a}}$ & Variably blue and red & Black/red & POS (magenta) & NEG \\
\hline $\begin{array}{l}\text { EmboGold }^{\mathrm{TM}} \\
\text { Microspheres }\end{array}$ & NEG (pink) & POS (red) & NEG (blue) & Variably blue and red & Black/red & POS (magenta) & NEG \\
\hline
\end{tabular}

${ }^{\mathrm{a}}$ The outer rim focally stains positive (pink/magenta).

This table contains a battery of special stains with results for cases 1-3 and the EmboGold ${ }^{\mathrm{TM}}$ Microspheres.

amounts of chronic inflammatory cells including lymphocytes, macrophages and multinucleated giant cells (Figures 4 and 5).
Only Case 1 was accompanied by a clinical history mentioning a previous fibroid embolization procedure. After characterization of the 'foreign 

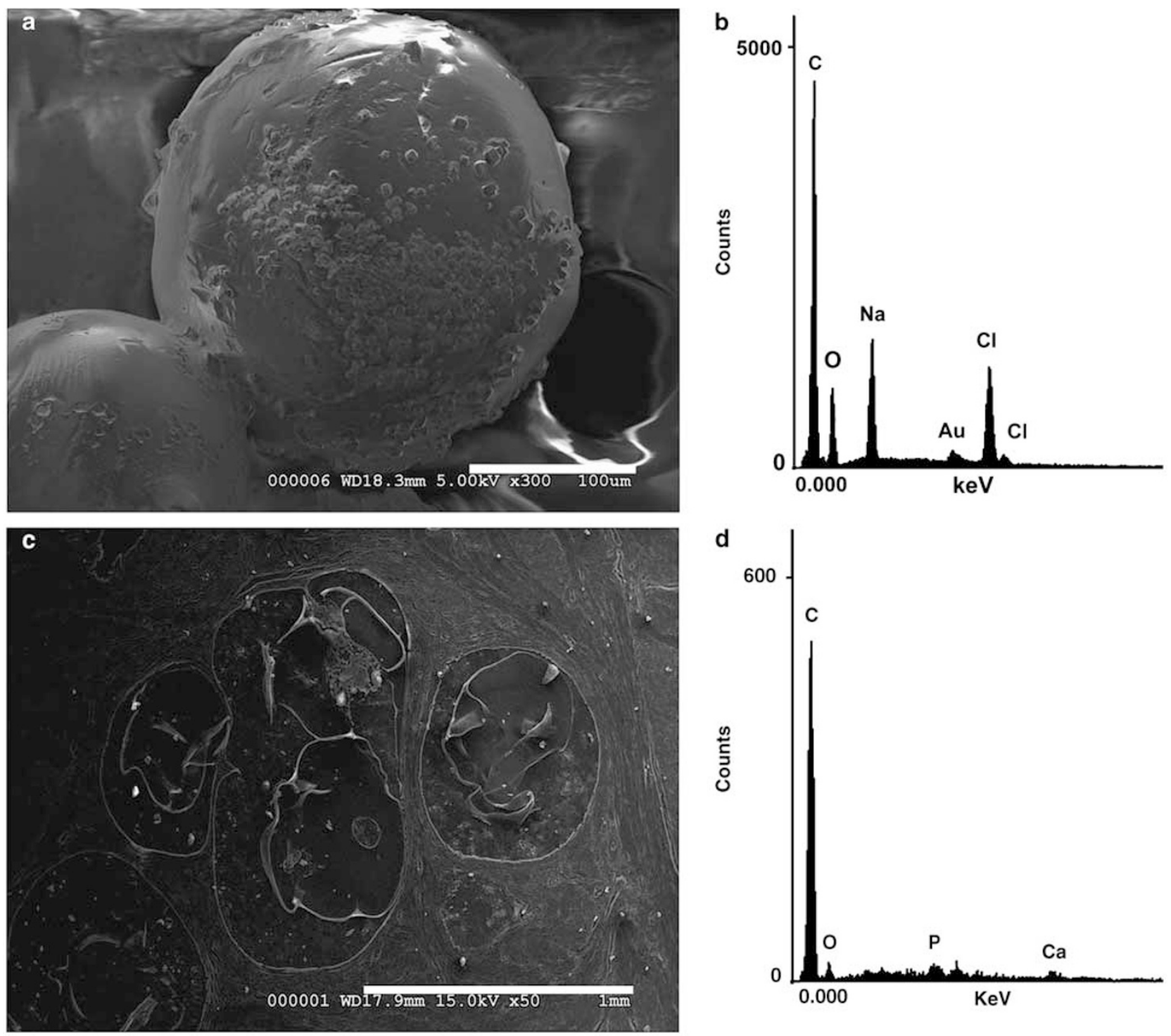

Figure 2 Scanning electron microscopy with energy dispersive X-ray analysis of the control material EmboGold ${ }^{\mathrm{TM}}$ Microspheres. (a, b) In vitro whole-mount EmboGold ${ }^{\mathrm{TM}}$ Microsphere shows the 500-700 $\mu \mathrm{m}$ microsphere with some shrinkage artifact from drying (approx. $350 \mu \mathrm{m}$ ), and contains elements: carbon, oxygen, sodium, gold and chlorine. (c, d) In vivo, uterine section of acrylic polyamide plastic embolization particles shows shatter 'Venetian blind effect' and contains elements: carbon, oxygen, phosphorus and calcium.

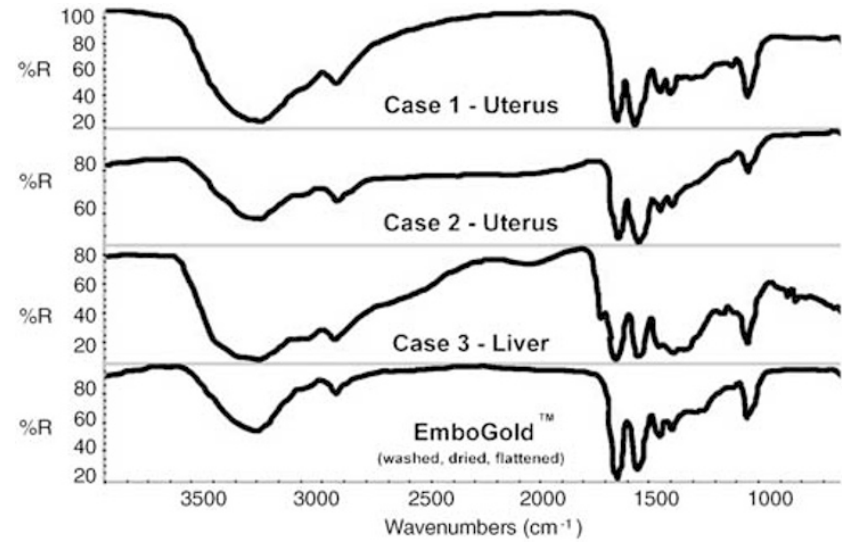

Figure 3 Infrared spectroscopy. Infrared spectra (cases 1-3) show wave numbers and peaks that match the reference acrylic polyamide plastic material of EmboGold ${ }^{\mathrm{TM}}$ Microspheres (bottom). material' in Case 2, and further questioning of the patient and clinician, a history of a uterine artery embolization 2 years prior to the hysterectomy was uncovered. The registered brand name of the embolization material was not included in either of the histories nor were we able to retrieve this information in any of the cases.

Embolized blood vessels in the liver (Figure 6) showed collections of rounded spaces, similar to those seen in the hysterectomy specimens, lined by foreign-body giant cells, nonbirefringent eosinophilic rounded particles with 'Venetian blind/fish scale' shatter artifact that lacked internal structures. Portal fibrosis with chronic inflammation, and scattered von Meyenburg complexes were also noted. A separate section taken from the mass showed a viable well-differentiated hepatocellular carcinoma. 

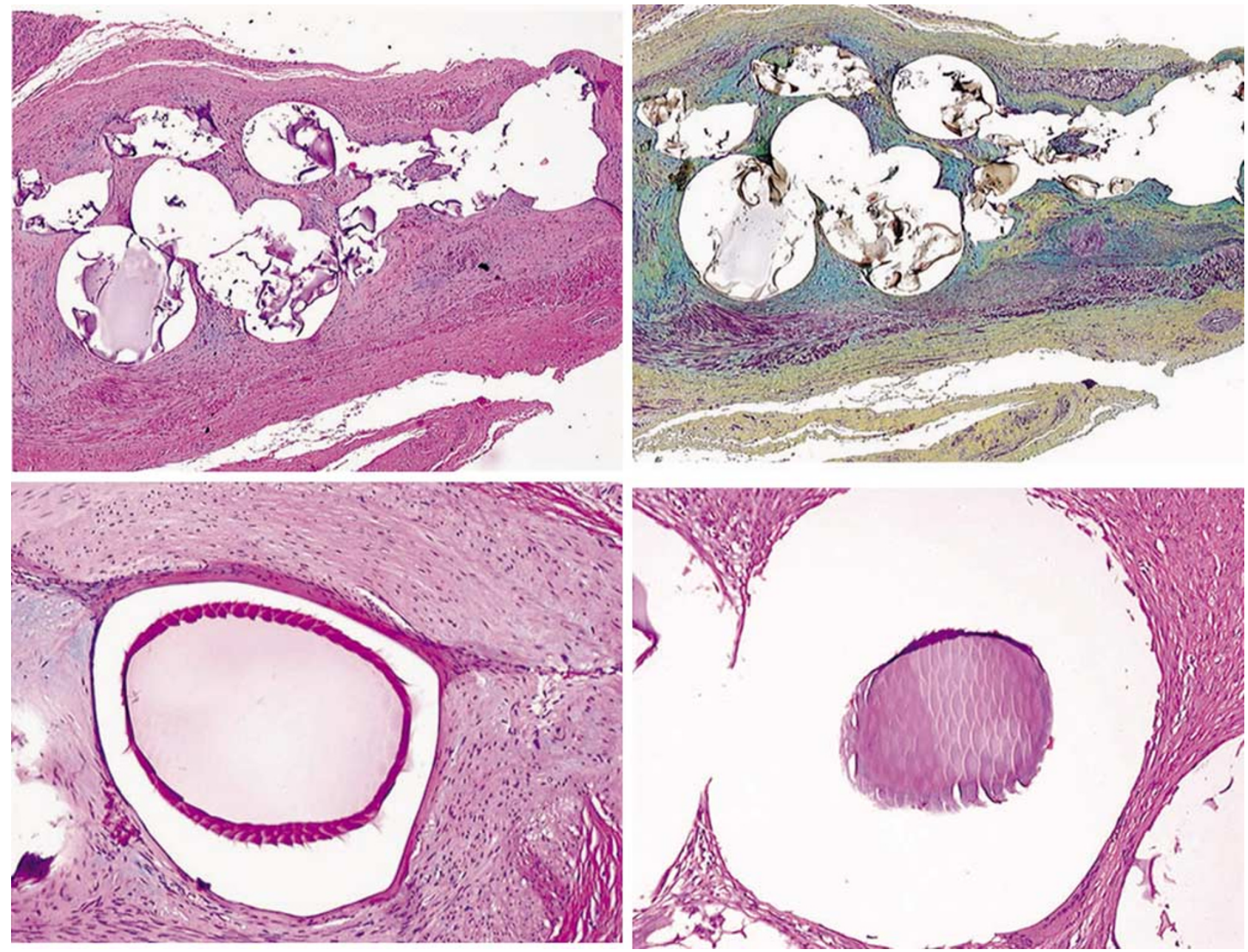

Figure 4 Case 1-uterus. Top: Uterine artery with obliterated lumen containing fibrous tissue, chronic inflammation and round spaces with remnants of embolization particles (hematoxylin and eosin stain, left; Pentachrome stain, right) $(\times 10$, both). Bottom: High-power view of embolized particles show the acrylic polyamide plastic (hematoxylin and eosin stains) $(\times 100$, both).

\section{Discussion}

Vascular embolization is a well-established practice for therapeutic treatment of various tumors and vascular lesions. We used Fourier transform infrared microscopy to analyze three specimens containing intravascular, round focally folded or distorted nonbirefringent unidentified material. All three cases exhibited similar infrared spectra, which closely resembled the spectrum obtained from the reference acrylic plastic polymer material (EmboGold ${ }^{\mathrm{TM}}$ ). This method of identification is highly accurate in correctly identifying unknown material such as an acrylic polyamide plastic, but does not identify the particular brand.

One of the limitations of infrared spectroscopy that applies to this study is that gelatin and tissue protein have similar spectra. This similarity emphasizes the value of having a suitable reference material (EmboGold $^{\mathrm{TM}}$ Microspheres in this case) for comparison.

We also used scanning electron microscopy with energy dispersive X-ray analysis to characterize the material seen. Scanning electron microscopy with energy dispersive X-ray analysis can give valuable information about the elemental composition of materials in tissue sections, for example, in the identification of silver in skin biopsies in patients with argyria. ${ }^{18,19}$ However, in this case, the elements identified (carbon and oxygen) are not helpful in identifying the material as they are ubiquitous in tissue, and calcium and phosphorus (usually as calcium phosphate), are commonly endogenous. In a chronic inflammatory process calcium phosphate deposition is considered to be a common nonspecific finding.

The scanning electron microscopy with energy dispersive X-ray analysis results from the reference material employed, EmboGold ${ }^{\mathrm{TM}}$ Microspheres, did not contain additional elements apart from sodium and chlorine (from saline, which surrounded the EmboGold $^{\mathrm{TM}}$ Microspheres in vitro), and gold. It is important to stress that the characterizations performed in these three cases indicates the presence of acrylic polyamide plastic microspheres, but does 

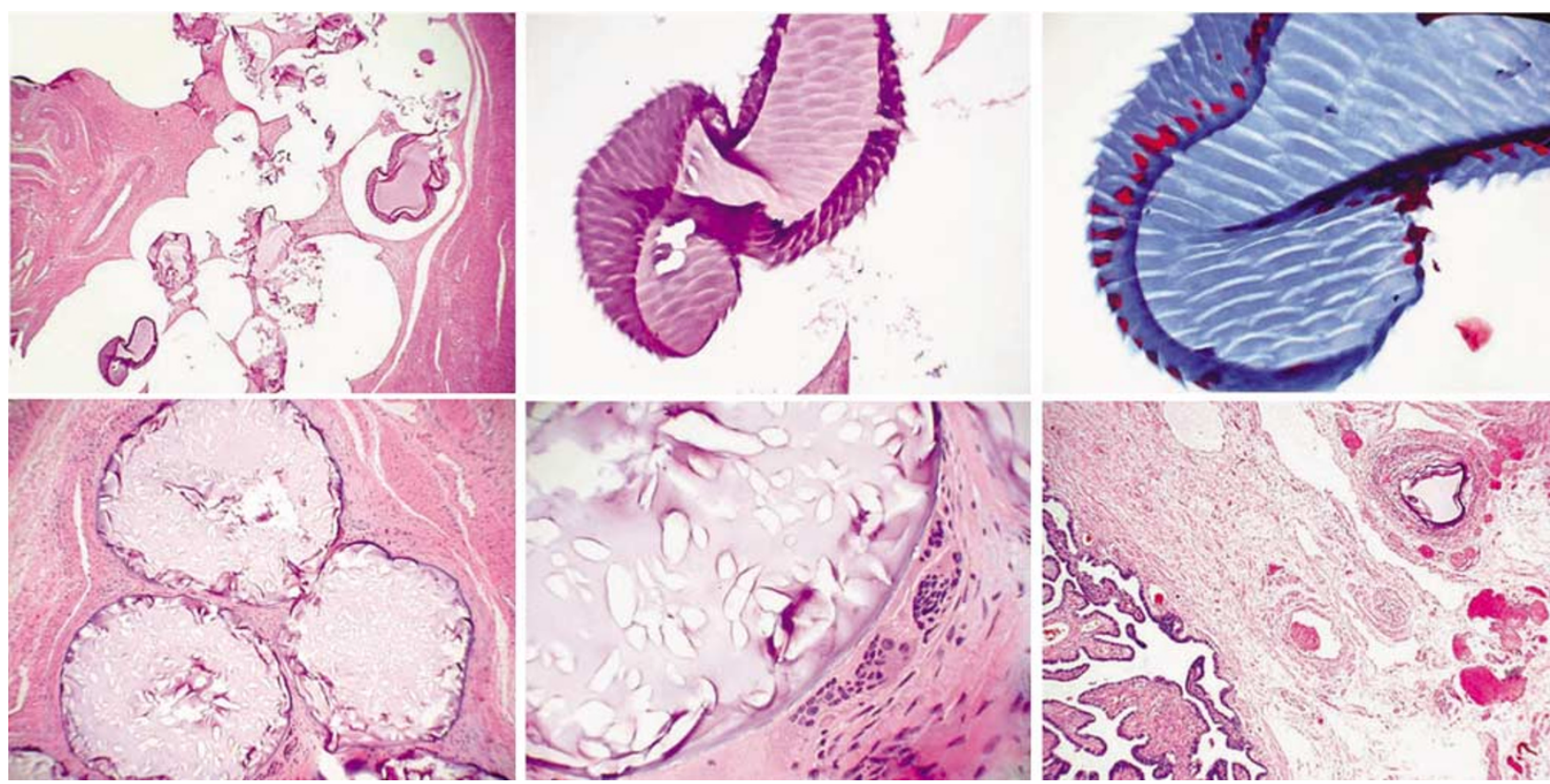

Figure 5 Case 2-uterus, ovary and fallopian tube. Top: Uterine artery with obliterated lumen shows fibrosis and round spaces containing remnants of embolized particles (left) $(\times 10)$. Higher power view of rounded spaces with embolization material (mid, hematoxylin and eosin stain; right, Masson's trichrome stain) ( $\times 100$ both). Bottom: Ovary (left and mid) and fallopian tube (right) arteries occluded with embolization particles. The connective tissue contains mild chronic inflammation and multinucleated foreign body giant cells (hematoxylin and eosin stains) $(\times 10$ left, $\times 100 \mathrm{mid} ; \times 5$ right).
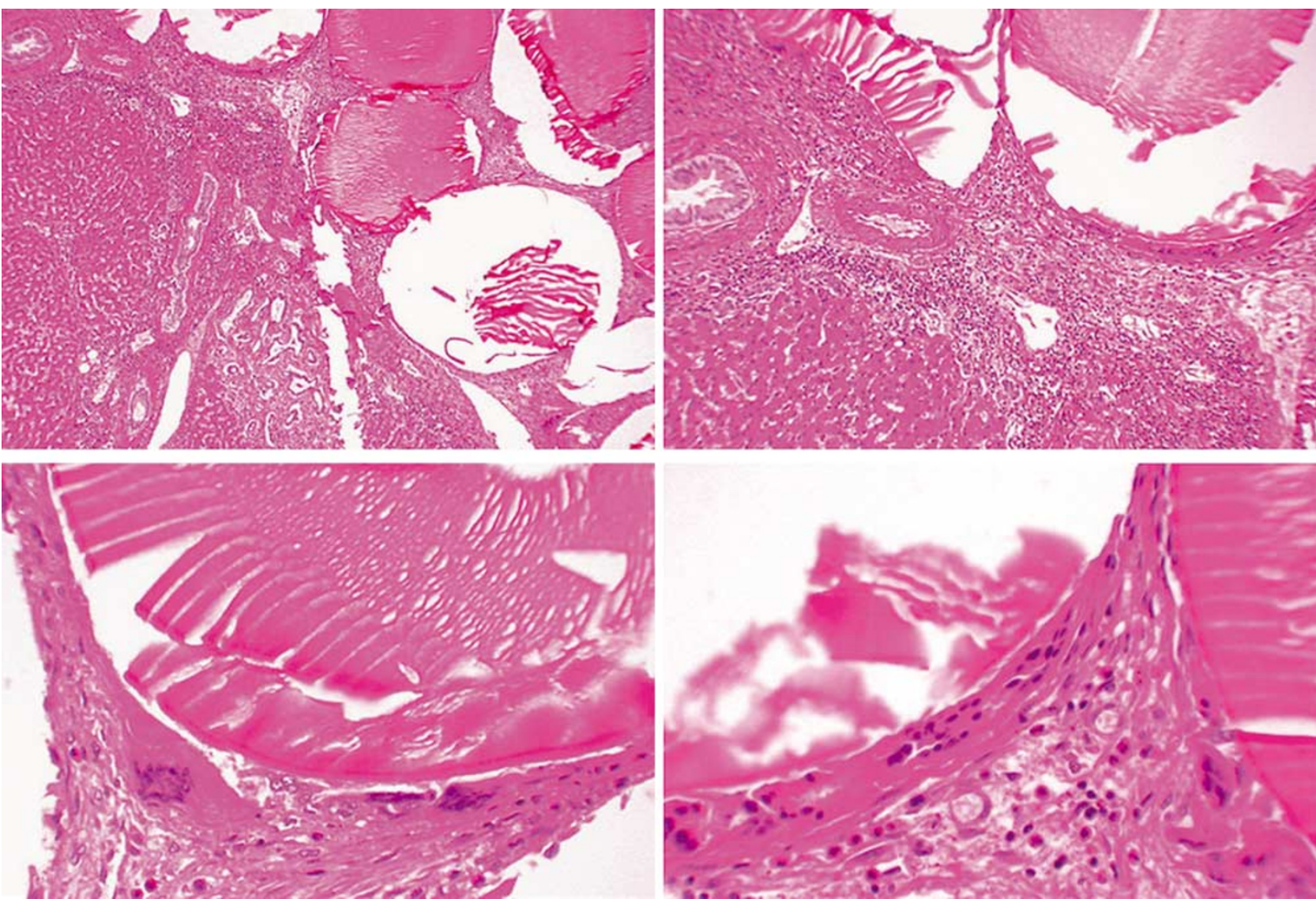

Figure 6 Case 3-liver. Top: portal tract with embolization particles showing shatter 'Venetian blind effect' artifact, chronic inflammation with foreign body giant cell reaction and a von Meyenburg complex. Bottom: Higher power view of foreign body giant cells adjacent to embolized particles (hematoxylin and eosin stains) $(\times 10$, upper left; $\times 50$, upper right; $\times 100$ lower left; under oil, $\times 250$ lower right). 
not identify the particular brand employed. In particular, gold was not detected in any of the three cases examined. The presence of gold may be somewhat specific to the reference material that was employed.

The criteria by which specific products are approved by the FDA vary from product to product. The reference material examined in vitro (EmboGold $^{\mathrm{TM}}$ Microspheres) is not approved for uterine artery embolization. However, it is approved for use in hypervascularized tumors and arteriovenous malformations (EmboGold ${ }^{\mathrm{TM}}$ product insert). The scanning electron microscopy with energy dispersive X-ray analysis finding of gold in an unidentified microsphere might be of some use in indicating the possible product used in future (nonuterine artery embolization) cases.

A disadvantage in using microspheres for embolization is the reported potential to pass through a lesion to the pulmonary circulation. ${ }^{20}$ It is therefore possible that pathologists will encounter embolization microspheres in locations remote from the intended site of intra-arterial placement.

Acrylic polyamide plastic embolization particles differ from helminths in that they have no internal structures. The remote possibility of observing helminths lacking visible internal structures may arise in areas of necrosis in which a worm has degenerated to an almost unrecognizable degree. However, in the three cases examined, the material was surrounded by fibrosis rather than necrosis, and although folded and showing cutting artifact the particles remained relatively well preserved, as might be expected for a nonresorbable material. ${ }^{20} \mathrm{In}$ two cases the interval between embolization and removal of the examined tissue was 2 years, and in the third case was unknown.

Given that sectioning of spherical objects may give particles with various diameters, and that some degree of shrinkage due to processing may occur, it is not unexpected that acrylic polyamide plastic embolization particles with listed diameters of up to $700 \mu \mathrm{m}$ may appear smaller in histological sections. There are several helminths that parasitize humans, which have diameters less than $700 \mu \mathrm{m} .{ }^{21}$ Another helpful feature is that the acrylic polyamide plastic embolization particles have circular or folded contours, whereas most worms are coiled in tissue and often demonstrate ovoid, longitudinal and circular contours.

Although most anatomic pathologists are familiar with the characteristics of commonly encountered surgical materials such as sutures, mesh, dental amalgam, silicone oil and gel foam, a number of more recently introduced materials are being encountered in tissue sections which can present a diagnostic challenge. Increased familiarity with the light microscopic features of these embolization particles and their intravascular location will enable pathologists to more rapidly and accurately recognize them in future specimens.
We believe that the lack of a prior clinical history of embolization at the time of initial evaluation in two of the three cases presented made the task of accurate interpretation of the observed histopathologic features more difficult. When faced with an unrecognized material in a histological section, a local pathologist may be able to access additional relevant clinical history relatively easily. As consultants, it is often relatively difficult for us to obtain such information. Interestingly, in the one case in which a history of prior embolization was given (Case 1), we were requested to rule-out 'a parasite' by the contributor. The broader issue in anatomic pathology of inadequate clinical information accompanying submitted specimens has possible detrimental effects including the performance of additional expensive studies. In a College of American Pathologists Qprobes study addressing the necessity of clinical information in surgical pathology, therapy-induced change was the disease process in which requested clinical information led to the highest proportion of changed diagnosis or revised reports. ${ }^{22}$

To our knowledge, this is the first report of the infrared spectra of acrylic polyamide plastic microsphere embolization products in human histologic tissue sections. In cases lacking appropriate clinical information, characterization by infrared microspectroscopy may be of considerable value to pathologists unfamiliar with this material's light microscopic appearance. In most cases, scanning electron microscopy with energy dispersive X-ray analysis does not add useful information to that obtained by infrared spectroscopy, but in problem cases it may he helpful to exclude the presence of other materials. Appropriate clinical history and the availability of a reference sample are valuable for accurate and timely characterization of unknown exogenous materials encountered in histopathological specimens.

\section{Acknowledgements}

We thank our colleagues at the Armed Forces Institute of Pathology and the contributors for their support. We also acknowledge the valuable work of Marie Jenkins, HT (ASCP) for scanning electron microscopy with energy dispersive X-ray analysis, and Ronald C Neafie, MS, Chief, Parasitic Diseases Pathology Branch, Armed Forces Institute of Pathology for valuable discussions.

The opinions or assertions contained herein are the private views of the authors and are not to be construed as official or as reflecting the views of the US Department of the Army, The Department of Defense, or the Department of Veterans Affairs.

\section{References}

1 Beaujeux R, Laureent A, Wassef M, et al. Trisacryl gelatin microspheres for therapeutic embolization, 
II: preliminary clinical evaluation in tumors and arteriovenous malformations. Am J Neuroradiol 1996;17:541-548.

2 Brown KT, Nevins AB, Getrajdman GI, et al. Particle embolization for hepatocellular carcinoma. J Vasc Intervent Radiol 1998;9:822-828.

3 Rand T, Loewe C, Schoder M, et al. Arterial embolization of unresectable hepatocellular carcinoma with use of microspheres, lipiodol, and cyanoacrylate. Cardiovasc Intervent Radiol 2005;28:313-318.

4 Ball DS, Heckman R, Olenick SW, et al. In vitro stability of tris-acryl gelatin microspheres in a multipharmaceutical chemoembolization solution. J Vasc Intervent Radiol 2003;14:83-88.

5 Misirli Y, Ozturk E, Kursaklioglu H, et al. Preparation and characterization of Mitomycin-C loaded chitosancoated alginate microspheres for chemoembolization. J Microencapsul 2005;22:167-178.

6 Weichert W, Denkert C, Gauruder-Burmester A, et al. Uterine arterial embolization with tris-acryl gelatin microspheres. A histopathologic evaluation. Am J Surg Pathol 2005;29:955-961.

7 Prollius A, deVries C, Loggenberg E, et al. Uterine artery embolization for symptomatic fibroids: the effect of the large uterus on outcome. Br J Obstet Gynaecol 2004;111:239-242.

8 Pelage J-P, Le Dref O, Beregi J-P, et al. Limited uterine artery embolization with tris-acryl gelatin microspheres for uterine fibroids. J Vasc Intervent Radiol 2003;14:15-20.

9 Spies JB, Benenati JF, Worthington-Kirsch RL, et al. Initial experience with the use of tris-acryl gelatin microspheres for uterine artery embolization of leiomyomata. J Vasc Intervent Radiol 2001;12: 1059-1063.

10 Spies JB, Ascher SA, Roth AR, et al. Uterine artery embolization for leiomyomata. Obstet Gynecol 2001; 98:29-34

11 Siskin GP, Englander M, Stainken BF, et al. Embolic agents used for uterine fibroid embolization. Am J Roentgenol 2000;175:767-773.

12 Siskin GP, Dowling K, Virmani R, et al. Pathologic evaluation of a spherical polyvinyl alcohol embolic agent in a porcine renal model. J Vasc Intervent Radiol 2003;14:59-98.

13 Laurent A, Beaujeux R, Wassef M, et al. Trisacryl gelatin microspheres for therapeutic embolization, I: development and in vitro evaluation. Am J Neuroradiol 1996;17:533-540.

14 Osuga K, Khankan AA, Hori S, et al. Transarterial embolization for large hepatocellular carcinoma with use of superabsorbent polymer microspheres: initial experience. J Vasc Intervent Radiol 2002;13:929-934.

15 Spies JB, Cooper JM, Worthington-Kirsch R, et al. Outcome of uterine embolization and hysterectomy for Leiomyomas: results of a multicenter study. Am J Obstet Gynecol 2004;191:22-31.

16 Prophet EB, Mills B, Arrington JB, et al. Armed Forces Institute of Pathology Laboratory Methods in Histotechnology. American Registry of Pathology: Washington, DC, 1992.

17 Luna LG. Microtomy artifacts. In: Histopathologic Methods and Color Atlas of Special Stains and Tissue Artifacts. Johnson Printers, Downers Grove: Illinois, 1992.

18 Landas S, Bonsib SM, Ellerbroek R, et al. Argyria: microanalytic-morphologic correlation using paraffinembedded tissue. Ultrastruct Pathol 1986;10:129-135.

19 Sato S, Sueki H, Nishijima A. Two unusual cases of argyria: the application of an improved tissue processing method for X-ray microanalysis of selenium and sulphur in silver-laden granules. Br J Dermatol 1999; 140:158-163.

20 Derdeyn CP, Graves VB, Salamat S, et al. Collagencoated acrylic microspheres for embolotherapy: in vivo and in vitro characteristics. Am J Neuroradiol 1997; 18:647-653.

21 Meyers WM, Neafie RC, Marty AM, Wear DJ (eds) Pathology of Infectious Diseases, Volume 1. Helminthiases. Armed Forces Institute of Pathology: Washington, DC, 2000.

22 Nakhleh RE, Gephardt G, Zarbo RJ. Necessity of clinical information in surgical pathology. A College of American Pathologists Q-Probes Study of 771475 surgical pathology cases from 341 institutions. Arch Pathol Lab Med 1999;123:615-619. 